\title{
Prevalence of Tension Type Headache among Young Adults and Their Gender Difference on Percieved Stress Scale: A Comparative Study
}

\author{
Priyanka Yadav ${ }^{1 *}$
}

\section{ABSTRACT}

Background: Tension-type headaches (TTH), together with migraines, are the most common primary headaches, affecting $80 \%$ of the general population. Stress is known to be a contributing factor to chronic tension-type headache (CTH), with research indicating that mental stress is the most commonly reported trigger and aggravating factor of a CTH episode. The study was conducted to find out the prevalence of TTH among youths of 18-25 age reporting frequent headache and to compare the perceived stress level among the diagnosed male and females of TTH. Methods: Perceived Stress Scale (PSS) rating and an IHS (International Headache Society) TTH Diagnostic questionnaire were used in this study. A sample of 150 students including 75 males and 75 females in the age group of 18-25 years complaining of frequent headache were taken from different colleges and universities located in Rewari district of Haryana. In the second phase, only the diagnosed cases of tension type headache participated in the study and fill the perceived stress scale questionnaire. After data collection, analysis of data using SPSS software was done which then further help in testing the hypothesis and extracting the result and inferences. Descriptive analysis of quantitative data expressed as mean and standard deviation. Mean and Chi square test were used for comparison of individual on quantitative parameters between groups. P value $<0.05$ was considered statistically significant. Results: The mean age of subjects with TTH was $22.79 \pm 2.14$. Prevalence of TTH among frequent headache sufferers is $68 \%$. Out of $68 \%$ TTH cases $66.7 \%$ subjects have episodic TTH and $33.3 \%$ have chronic TTH. Value of PSS * Gender Pearson chi square is 5.151 at a significance value of .076 and it shows that there would be no significant differences exist between males and females on perceived stress score. Conclusion: TTH is more prevalent among females as compared to males. No significant differences exist between males and females on perceived stress scores.

Keywords: Tension Type Headache, Young Adults, Gender Difference, Percieved Stress Scale

\footnotetext{
${ }^{1}$ Research Scholar, GKV, H.N-13, Near pump house, Rewari, Haryana, India *Responding Author

(C) 2016 Yadav P; licensee IJIP. This is an Open Access Research distributed under the terms of the Creative Commons Attribution License (http://creativecommons.org/licenses/by/2.0), which permits unrestricted use, distribution, and reproduction in any Medium, provided the original work is properly cited.
} 


\section{Prevalence of Tension Type Headache among Young Adults and Their Gender Difference on Percieved}

Stress Scale: A Comparative Study

Tension type headache (TTH) is the most common form of headache what many people consider a normal headache in contrast to migraine. The recent second version of the International headache society classification distinguishes between three forms of TTH primarily on basis of headache frequency: 1) in frequent episodic TTH (fewer than 12 headache days/year). 2) frequent episodic TTH (between 12 and 180 days/year). 3) chronic TTH (at least 180 days/year) (Ashina, S.; Bendtsen, L; Jensen, R., 2006). TTH is characterized by a bilateral, pressing, tightening pain of mild to moderate intensity, occurring in short episodes of variable duration (episodic forms) or continuously (chronic forms). The headache is not associated with typical migraine features such as vomiting, severe photophobia and phonophobia. In the chronic form only one of these accompanying symptoms is allowed and only mild nausea is accepted (Jensen, R., 1999). Stress is the non-specific response of the body to any demand for change (Seyle, H., 1936). Stress is known to be a contributing factor to chronic tension-type headache (CTH), with research indicating that mental stress is the most commonly reported trigger and aggravating factor of a CTH episode (Cathcart S.et al. 2010; Olesen J. 1991). One hypothesis for the mechanism by which stress contributes to CTH is that it aggravates already increased pain sensitivity in CTH sufferers. Correlations among pain sensitivity, stress and headache activity, and evidence that both stress and pain sensitivity predict headache activity, support this central model (Cathcart, S. et al. 2008; Bottos, S. et al. 2004). Furthermore, experimentally inducing mental stress has been demonstrated to increase pain sensitivity and headache intensity in CTH sufferers.

Published estimates of prevalence of TTH vary over a wide range from $1.3 \%$ to $65 \%$ in men and $2.7 \%$ to $86 \%$ in women. Nine studies have used the widely accepted 1988 IHS criteria to assess the epidemiology of TTH, but even among these studies prevalence estimates vary widely. Prevalence increased with increasing education level in both sexes, reaching a peak in subjects with graduate education of $48.5 \%$ for men and $48.9 \%$ for women (Brian, S.S.; Walter, F. S.; David, S.; Richard, B.L., 1998).

Young subjects are more frequently affected than older subjects (Jensen, R., 1999). The femaleto -male ratio of TTH is 5:4 indicating that, unlike migraine, women are affected slightly more than men (Stovner, L; Hagen, K. et. al.,2007). Tension-type headache is more prevalent in females than in males (male: female ratio about 1:1.5), and in both sexes prevalence declines with age (Philips 1977; Abramson et al. 1980; Rasmussen et al. 1991; Pryse-Phillips et al. 1992). Any comparison of prevalence between populations must take into account age and sex differences. On average, perceived stress is higher in individuals who report chronic daily headache than in a healthy population. Although recurrent TTH sufferers have similar physiological responses to laboratory stressors, they report a greater number of everyday stresses or daily "hassles" than do matched non-headache control subjects. Furthermore, stressful events are appraised as more stressful for recurrent TTH sufferers than for headache-free subjects.

(C) The International Journal of Indian Psychology, ISSN 2348-5396 (e) I ISSN: 2349-3429 (p) | 172 
Stress is also the most frequent headache trigger with $88 \%$ of patients from the general population in urban and rural areas reporting this variable as a cause for ensuing headache. The prevalence peaks between ages 30 to 39 and decreases slightly with age. Risk factors for developing TTH were poor self- rated health, inability to relax after work, and sleeping less hours per night (Lyngberg, A.C; Rasmussen, B.K. et. al., 2005).

Headaches generally are reported to occur in relation to emotional conflict and psychosocial stress, but the cause-and-effect relationship is not clear. The triggers reported most frequently for TTH are stress (mental or physical), irregular or inappropriate meals, high intake of coffee and other caffeine containing drinks, dehydration, sleep disorders, too much or too little sleep, reduced or inappropriate physical exercise and psychologic problems, (Rasmussen, B.K.; Jensen, R.;Schroll, M. et.al., 1992). The combination of non-pharmacologic and pharmacologic treatments are prescribed separately but should go hand in hand. Psychological treatment strategies have reasonable scientific support for effectiveness. Relaxation training is a selfregulation strategy that provides patients with the ability to consciously reduce muscle tension and autonomic arousal that can precipitate and result from headaches. It is most likely that cognitive changes (i.e self-efficacy) rather than reductions in muscle tension account for the improvement in TTH with EMG (electromyography) biofeedback.

Cognitive-behavioural therapy (stress management) aims to teach patients to identify thoughts and beliefs that generate stress and aggravate headaches. The exact degree of effect of psychological treatment strategies is difficult to estimate but Cognitive behavioural therapy is as effective as tricyclics antidepressants, whereas a combination of the two treatments seemed more effective than either treatment alone. Research indicates that CBT and stress management is most effective when combined with relaxation training or biofeedback (Holroyd, K.A.; Donnell, F.J., et.al., 2001). Finally, stress has been noted to exacerbate headache symptoms; and minor everyday stressors, rather than major life events, have been tagged as a contributor to maintaining or prolonging existing headache. Thus, stress, particularly as a result of minor everyday frustrations, is an important area of investigation in relation to headache.

Most of the evidences on Tension type headache and comorbid stress disorder comes from western studies. There is very little research looking into the prevalence of this psychological disorder in patients with TTH in a developing country like India. Due to significant sociocultural differences, it is difficult to generalize research findings from developed countries. This therefore warrants a need to undertake basic research in a developing country like India. This study is conducted with the aim to study the prevalence of tension type headache in male /female students suffering from frequent headaches. And to compare the perceived stress level among the diagnosed male and females of TTH. 


\section{MATERIALS AND METHODS}

\section{Sample and Data Collection}

A sample size of 150 students including 75 males and 75 females in the age group of 18-25 years complaining of frequent headache was taken from different colleges and universities located in Rewari district of Haryana. The primary method of data collection was self-report questionnaires. The PSS rating and IHS TTH Diagnostic questionnaire were used in this study. The Perceived Stress Scale (PSS10) was used to measure the degree to which situations in one's life are appraised as stressful (Cohen \& Williamson, 1988).

\section{Inclusion criteria}

Consented subjects of 18-25 years should report frequent headaches, should not currently receiving (or having received in the past 12 months) intervention for headache. No psychiatric or major medical condition currently or in the past 12 months, no concurrent headache or pain symptoms or diagnoses other than CTH. CTH subjects were required to not be taking or not have taken in the past three months any analgesic medication other than $\leq 1000 \mathrm{mg}$ per day of acetylsalicylic acid or paracetamol. None of the subjects reported taking prophylactic medication for headache or daily analgesic use.

\section{Tools and Techniques}

IHS (International Headache Society) Headache Diagnostic Questionnaire (Fishbain, D.A. et.al., 2001) to diagnose the tension type headache. It has satisfactory reliability and validity. The International Headache Society classification of primary headaches in the International Classification of Headache Disorders (ICHD) is almost universally accepted by researchers and clinicians. It is highly unlikely that reputable journals will accept submissions for publication if the cohorts have not been selected strictly according the ICHD. Likewise, in the clinical setting the appropriate treatment is prescribed according to how the patient's headache is classified.

Perceived Stress Scale- Perceived Stress Scale (PSS) was developed by Cohen (1985). It is designed to measure the degree to which respondents found their lives "unpredictable, uncontrollable, and overloading" (Cohen \& Williamson, 1988, p. 34). The scale also includes a number of direct queries about current levels of experienced stress. As a result of factor analysis, a shorter version of the PSS scale was developed (Cohen and Williamson 1988) by the authors of the original PSS. The PSS10 was derived by dropping four items from the original scale. Cronbach's alpha coefficient for the PSS10 was .78. Individual scores on the PSS can range from 0 to 40 with higher scores indicating higher perceived stress. Scores ranging from $0-13$ would be considered low-stress. Scores ranging from 14-26 would be considered moderate stress. Scores ranging from 27-40 would be considered high perceived stress levels. The Perceived Stress Scale has been shown to have a high degree of reliability and validity. 
Prevalence of Tension Type Headache among Young Adults and Their Gender Difference on Percieved Stress Scale: A Comparative Study

\section{Procedure}

Subjects complaining frequent headaches and fulfilled the inclusion criteria of the study were asked to fill IHS Headache Diagnostic questionnaire and then assessed to diagnose the possible cases of tension type headache. In the second phase, only the diagnosed cases of tension type headache participated in the study. Those subjects then fill the perceived stress scale questionnaire. After collecting the sufficient data, the next step was the analysis of data which then further help in testing the hypothesis and extracting the result and inferences.

\section{Statistical Analysis}

The analysis of collected data included differentiating the subjects on the basis of TTH criteria into two groups based on their gender. Descriptive analysis of quantitative data expressed as mean and standard deviation. Qualitative data were expressed as percentage and absolute numbers. Mean and Chi square test were used for comparison of individual on quantitative parameters between groups using SPSS software. P value $<0.05$ was considered statistically significant.

\section{RESULTS}

The mean age of subjects with TTH was $22.79 \pm 2.14$. Out of 150 headache patients, only 102 patients were diagnosed with tension type headache, in which 42 were males and 60 were females and these 102 subjects then rated on PSS. Male/female distribution was 50:50 in subjects reported frequent headache group. Male/female distribution was then left with 28: 40 in TTH group and subjects rated on PSS.

\section{PSS * Gender Cross-tabulation}

\begin{tabular}{|c|c|c|c|c|c|}
\hline & \multicolumn{2}{|c|}{ Gender } & \multirow[t]{2}{*}{ Total } \\
\hline & & & Male & Female & \\
\hline \multirow{6}{*}{ PSS } & \multirow{2}{*}{ Average Stress } & Count & 5 & 1 & 6 \\
\hline & & \% within PSS & $83.3 \%$ & $16.7 \%$ & $100.0 \%$ \\
\hline & \multirow{2}{*}{ High Stress } & Count & 19 & 26 & 45 \\
\hline & & \% within PSS & $42.2 \%$ & $57.8 \%$ & $100.0 \%$ \\
\hline & \multirow{2}{*}{ Very High Stress } & Count & 18 & 33 & 51 \\
\hline & & \% within PSS & $35.3 \%$ & $64.7 \%$ & $100.0 \%$ \\
\hline \multirow{2}{*}{\multicolumn{2}{|c|}{ Total }} & Count & 42 & 60 & 102 \\
\hline & & \% within PSS & $41.2 \%$ & $58.8 \%$ & $100.0 \%$ \\
\hline
\end{tabular}

\section{Table 1}

The prevalence of TTH among frequent headache sufferers was found to be $68 \%$. Out of 102 diagnosed cases of TTH, 68 fulfill the criteria of episodic TTH and 34 are diagnosed with chronic TTH. It means 66.7\% subjects are having episodic TTH and 33.3\% have chronic TTH. On statistical analysis it was found that there is no significant difference among males and

(c) The International Journal of Indian Psychology, ISSN 2348-5396 (e)| ISSN: 2349-3429 (p) | 175 


\section{Prevalence of Tension Type Headache among Young Adults and Their Gender Difference on Percieved}

Stress Scale: A Comparative Study

females on Perceived Stress Score. That is females show high prevalence of TTH as compared to males and scores of both males and females are almost same on PSS.

Above table 1 represents the cross-tabulation of PSS and Gender data. It indicates the stress level of males and females irrespective of the type of headache they are suffering from. The frequency and percentage of such males and females is indicated in the table. It shows that out of total 102 diagnosed cases of TTH, 42 (41.2\%) males and 60 (58.8\%) females reported on stress scale. Out of total $42(41.2 \%)$ males, 5 (83.3\%) males scored as average stress, 19 (42.2\%) males scored as high stress and 18 (35.3\%) males scored as very high stress.

Likewise males, females are too scored on stress scale. Out of total 60 (58.8\%) females, 1 (16.7\%) female scored as average stress, 26 (57.8\%) females scored as high stress and 33 (64.7\%) females scored as very high stress.

Chi-Square Tests

\begin{tabular}{|l|l|l|l|}
\hline & Value & Df & Asymp. Sig. (2-sided) \\
\hline Pearson Chi-Square & 5.151 & 2 & .076 \\
\hline Likelihood Ratio & 5.289 & 2 & .071 \\
\hline Linear-by-Linear Association & 3.365 & 1 & .067 \\
\hline N of Valid Cases & 102 & & \\
\hline
\end{tabular}

\section{Table 2}

Above table 2 shows the chi square values related to PSS * Gender Cross-tabulation keeping p value $<0.05$ as significant. Value of PSS * Gender Pearson chi square is 5.151 at a significance value of .076 and is considered as non-significant differences exists in the relationship of PSS * Gender. It means that the difference seems to exist in the PSS level among males and females are non-significant that is stress scores of males and females with TTH are approximately the same. It shows that there would be no significant differences exist between males and females on perceived stress score. So, our hypothesis is rejected on the basis of chi-square value $(\mathrm{p}<0.05)$.

\section{DISCUSSION}

Chronic headache is as much as a problem in India as elsewhere in the world with a rising trend in young adults which negatively affects the quality of life of the affected person. In current scenario of increasing prevalence of headache in students, most of them have been found to practice self -medication leading to inappropriate management and sometimes analgesic overuse causing treatment refractoriness. Headache patients frequently report stress to be one of their main activators or aggravators for headache. The association between stress and TTH has been stressed by many authors. It is supposed that stress can be a predisposing factor contributing to

(c) The International Journal of Indian Psychology, ISSN 2348-5396 (e)| ISSN: 2349-3429 (p) | 176 
the onset of headache disorders, that it accelerates the progression to chronic headache, provokes and exacerbates headache episodes and that the headache experience itself can serve as a stressor. It is also revealed that subjects who are diagnosed with TTH of either type also have higher stress scores.

Sara, H. et al. 2014 studied the association between stress intensity and headache frequency for tension-type headache (TTH), migraine and migraine with coexisting TTH (Migraine TTH). a population-based sample of 5159 participants (21-71 years) who were asked quarterly between March 2010 and April 2012 about headache and stress. Log-linear regression in the framework of generalized estimating equations was used to estimate regression coefficients presented as percent changes to describe the association between stress intensity (modified visual analog scale (VAS) from 0 to 100) and headache frequency (days/month) stratified by headache subtypes and age groups and adjusted for sex, age, frequent intake of acute pain drugs, drinking, smoking, BMI and education. That study provides evidence for an association between stress intensity and headache frequency. It is found that participants who reported headache experienced more stress compared to participants without headache. Increasing stress was associated with increasing headache frequency for all headache subtypes, which was particularly pronounced in participants with TTH and younger headache sufferers. Women and men reported almost the same mean stress level.

Behavioral treatments (i.e. cognitive behavioral therapy, biofeedback (Andrasik, F. 2010), relaxation training) have demonstrated clinical efficacy when practiced correctly. Yoga are mentioned to improve headache as well (Campbell, J. K. et al. 2008). Behavioral treatments may be used individually or in conjunction with pharmacologic and other interventions and may augment the effectiveness of other treatments, or minimize the need for their use. Our results underline the need for stress management strategies in people with chronic headache or having a high or with very high scores.

\section{Acknowledgments}

The author appreciates all those who participated in the study and helped to facilitate the research process.

\section{Conflict of Interests}

The author declared no conflict of interests.

\section{REFERENCES}

Abramson, J.H., Hopp, C., Epstein, L.M. (1980). Migraine and non-migraine headaches. A community survey in Jerusalem. J Epidemiol Community Health; 34:188-193. 
Prevalence of Tension Type Headache among Young Adults and Their Gender Difference on Percieved Stress Scale: A Comparative Study

Andrasik, F. (2010). Biofeedback in headache: An overview of approaches and evidence. Cleve Clin J Med, 77(Suppl 3),72-76.

Ashina M. Neurobiology of chronic tension-type headache. Cephalalgia 2004; 24(3):161-72.

Bendtsen, L., Jensen, R. (2006). Tension-type headache: the most common, but also the most neglected, headache disorder. Curr Opin Neurol;19(3):305-9.

Bottos, S., Dewey, D. (2004). Perfectionists appraisal of daily hassles and chronic headache. Headache, 44,772-9.

Brian, S., Walter, F., Stewart, Simon, D., Richard, B., Lipton. (1998). Epidemiology of tension type headache. JAMA. 279, 381-383.

Campbell, J.K., Penzien, D.B., Wall, E.M. (2008). Evidence-based guidelines for migraine headache: Behavioral and physical treatments. US Headache Consortium 2000.

Cathcart, S., Winefield, A.H, Lushington, K., \& Rolan P. (2010) Stress and tension-type headache mechanisms. Cephalalgia, 30, 1250-67.

Cohen, S., Kamarck, T.,\& Mermelstein, R. (1983). The PSS Scale : A global measure of perceived stress. Journal of Health and Social Behavior. American Sociological Association, 24, 386-396.

Cohen, S., Wills. (1988). Stress, social support and the buffering hypothesis. Psychological Bulletin, 98, 310-357.

Fishbain, D. A., Cutler, R., Cole, B., Rosomoff, H. L.,\& Rosomoff, R. S. (2001). Clinical Journal of Pain, 17(1), 78-93.

Headache Classification Subcommittee of the International Headache Society. (2004). The international classification of headache disorders. (2nd edition). Cephalalgia, 24 (1), 1-160.

Holroyd, K. A., O’Donnell, F. J., \& Stensland, M. (2001). Management of chronic tension type headache with tricyclic antidepressant medication, stress management therapy, and their combination: a randomized controlled trial. Journal Of American Medical Associations, 285(17), 2208-15.

Holroyd, K.A, Stensland, M., Lipchik, G.L., Hill, K.R., O'Donnell, F.S., Cordingley, G. (2000). Psychosocial correlates and impact of chronic tension-type headaches. Headache. 40, 3-16.

Holroyd, K.A., O'Donnell, F.J., Stensland, M., Lipchik, G.L., Cordingley, G.E., Carlson, B.W. (2001). Management of chronic tension-type headache with tricyclic antidepressant medication, stress management therapy, and their combination: A randomized controlled trial. JAMA. 285, 2208-2215.

Lyngberg, A.C., Rasmussen, B.K., \& Jorgensen, T. (2005) Prognosis of migraine and tensiontype headache: population-based follow-up study. Neurology, 65(4), 580-5.

Olesen, J. (1991) Clinical and pathophysiological observations in migraine and tension-type headache explained by integration of vascular, supra-spinal and myofascial inputs. Pain, 46, 125-32.

Philips, C. (1977). Headache in general practice. Headache; 16:322-329. 
Pryse-Phillips, W., Findlay, H., Tugwell, P., et al. (1992). Canadian population survey on the clinical, epidemiologic and societal impact of migraine and tension-type headache. Can $J$ Neurol Sci; 19:333-339.

Rasmussen, B.K., Jensen, R., Schroll, M., Olesen, J. (1991). Epidemiology of headache in a general population: a prevalence study. J Clin Epidemiol 44:1147-1157.

Rasmussen, B.K. (1993). Migraine and tension-type headache in a general population: Precipitating factors, female hormones, sleep pattern and relation to lifestyle. Pain. 53, 65-72.

Schramm, S.H., Moebus,S., Lehmann, N., Bock, E. et.al (2014). The association between stress and headache: A longitudinal population-based study. Cephalalgia.

Seyle, H. (1936). Definition of stress. The American Institute of Stress.

Stovner, L., Hagen, K., Jensen, R., et al. (2007). The global burden of headache: A documentation of headache prevalence and disability worldwide. Cephalalgia. 193-210.

How to cite this article: Yadav P (2016), Prevalence of Tension Type Headache among Young Adults and Their Gender Difference on Percieved Stress Scale: A Comparative Study, International Journal of Indian Psychology, Volume 4, Issue 1, No. 80, ISSN:2348-5396 (e), ISSN:2349-3429 (p), DIP:18.01.118/20160401, ISBN:978-1-365-57867-0 